Sri Lankan Journal of Anaesthesiology 17(2) : 65 - 68 (2009)

\title{
SUPRACLAVICULAR SUBCLAVIAN VEIN CATHETERIZATION IN MORBIDLY OBESE
}

\author{
Chaminda Pathiraja ${ }^{1 *}$, Graham Sharpe ${ }^{2}$, Jacek Woojner ${ }^{3}$, Richard Stubbs ${ }^{4}$ \\ 1, 2, 3, 4, Wakefield G.E Centre, Wellington, New Zealand \\ *Corresponding author: chamindapathi@yahoo.com
}

Key words: cannulation, venous

\begin{abstract}
Background:
Obesity is one reason which will make central venous catheterization a difficult procedure. Ultrasound will help in locating the veins but in some patients because of short neck, internal jugular approach will be technically difficult. Supraclavicular approach to subclavian cannulation has been described as being less dependent on anatomical land marks and associated with comparatively less complications even in inexperienced hands. Literature does not show the effectiveness of this method in morbidly obese patients. Our aim of this study was to investigate the rates of success and complications of supraclavicular subclavian central venous catheterization in the morbidly obese.
\end{abstract}

\section{Methods:}

From amongst the morbidly obese patients who presented for FOBI gastric pouch bypass surgery, supraclavicular approach to subclavian venous cannulation was attempted by an anaesthetic registrar who was relatively new to this technique. Closed questionaire was used to gather data such as age, gender, weight, height, BMI, attempts with seeker needle and proper needle. Immediate and late complications were noted till catheter removal.

\section{Results:}

Catheterization was attempted in 48 patients with mean weight $131.29+/-29.89 \mathrm{~kg}$ and mean BMI of 47.60+/-9.80. Overall success rate was $97.9 \%$ (47 out of 48 patients) with average attempts 1.44 (68 in 47 successful cannulations) of seeker needle and with average attempts 1.40 (66 in 47 successful cannulations) of proper needle. There was 01 pneumothorax, 01 catheter malposition and 01 catheter related infection.

\section{Conclusion:}

Supraclavicular approach is a reliable way of obtaining central venous access with less complications in obese, even in less experienced hand.

\section{Introduction}

Central venous catheterization is often performed for fluid infusion in patients with poor peripheral access, haemodynamic monitoring administrate only meant to be given via a central line and infusion of irritable or hypertonic solutions and for haemodialysis.

In our institute we placed central venous catheters in gastric bypass patients during the anesthetic as a reliable venous access to use during the perioperative period. They need fluids and routine blood investigations during the first few postoperative days. The catheter is removed on the $6^{\text {th }}$ postoperative day if patient did not encounter any complications. Occasionally it is used to observe haemodynamic status. Aubaniac first described an approach to subclavian vein cannulation in 1952. ${ }^{(1)}$ Subsequently various methods have been described to gain central venous access. Most of them rely on anatomical land marks. With the introduction of ultra sound imaging for central venous catheterization, 
multiple investigations document that ultrasound guidance of central venous catheterization is associated with enhanced ease of catheter insertion and fewer complications. ${ }^{(7)(15)}$ Majority of our obese patients anatomical land marks are not well demarcated in the neck and movement reduced with a short neck making it difficult to use internal jugular approach. Mixed results are reported with the use of ultrasound during subclavian vein catheterization. ${ }^{(12)}$ This is probably because the subclavian vein is not as well visualized as the internal jugular vein. Therefore we used either supra clavicular or infraclavicular approach to subclavian vein using anatomical land marks to get central venous access. Literature indicates relatively easy learning curve for supraclavicular approach in the general population and our objective in this study was to investigate the rates of success and complications of supraclavicular subclavian central venous catheterization in the obese.

\section{Method}

All the patients who had FOBI gastric bypass surgery during the period from $1^{\text {st }}$ September to $28^{\text {th }}$ February were included in this study. Any other central venous approaches or supra clavicular approach by a consultant were excluded. Only attempts made by an anaesthetic registrar who did not have previous exposure to supraclavicular approach were considered.

A questionnaire containing closed questions was used to collect data on the following variables. Age, weight, height ,sex, attempts with the seeker and proper needle, success/failure ,complications and treatment of complications. Each skin puncture was defined as an attempt. The following were defined as complications related to the insertion of the catheter; pneumothorax, haemothorax, hydrothorax, diaphragmatic paralysis, arterial puncture, bleeding and haematoma at the point of insertion. Chest $x$ rays were taken in the recovery room to assess catheter position and the occurrence of complications. Catheter site was inspected for any evidence of infection and catheter was removed on the $6^{\text {th }}$ postoperative day if patient did not encounter any complications.

\section{Procedure}

The patient was placed in a supine, 15 degree trendelenburg position with head slightly rotated towards the opposite side .All were given standard general anesthetic and mechanically ventilated with out " peep" during the procedure. Right/supraclavicular fossae including the suprasternal notch was cleaned with alcohol. Sterile technique was used by the operator. Whenever possible the lateral border of the sternocleidomastoids just above the clavicle was used as the insertion point. When it is not well demarcated, junction between medial and middle third of the clavicle was used as the insertion point. A 22 gauge long seeker needle was inserted bisecting the sterno cleidomastoidclavicular angle with 10 degrees above the horizontal plane, which was similar to the technique describe by Yoffa. ${ }^{(17)}$ After each pass the barrel of the syringe was elevated a few degrees. Each attempt was made slowly with gentle aspiration applied continuously. If the first pass was unsuccessful the subsequent attempts were performed by bringing the needle to a subcutaneous position and re entering at a new angle. After locating the site of the vein with the seeker, direction and the depth was marked and proper needle was inserted. Modified Seldinger technique was used to place the catheter. Once guide wire was in place whenever possible we railroaded a long 18 gauge cannula to confirm venous placement and reintroduced the guide wire "J" tip making it facing upwards. By pulling the guide wire proximally and fixing it distally will straighten the " $\mathrm{j}$ " tip making " $\mathrm{J}$ " for easy reintroduction. Then catheter was introduced and flushed with normal saline after aspiration. Fixing was done with nylon sutures and sterile dry dressing was used to cover the insertion point.

\section{Results}

Total of 61 patients were operated for FOBI gastric bypass surgery during a period of 6 months.12 patients either had other approaches or consultant attempts to place the central venous catheter. In 48 patients supra clavicular approach was attempted by the anaesthetic registrar to achieve central venous cannulation. 39 were females and 9 were males. 
Demographic details of the study population Table 1

\begin{tabular}{|l|l|l|l|c|}
\hline & Max & Min & Average & SD \\
\hline Age & 70 & 20 & 44.85417 & $+/-11.98608$ \\
\hline $\begin{array}{l}\text { Height } \\
(\mathrm{kg})\end{array}$ & 153 & & 65.9167 & $+/-6.531441$ \\
\hline $\begin{array}{l}\text { Weight } \\
\text { (m) }\end{array}$ & 267 & 94 & 131.2917 & $+/-29.8932$ \\
\hline BMl & 86.2 & 5.6 & 47.60333 & $+/-9.802987$ \\
\hline
\end{tabular}

Success and failure rates and attempts to success

Table 2

Failure rate $-2.1 \%$ ( 1 in 48$)$

Success rate $-97.9 \%$ (47 in 48 )

\begin{tabular}{|l|l|l|}
\hline \multicolumn{2}{|l|}{$\begin{array}{l}\text { Attempts with seeker needle } \\
\text { average } 1.44 \\
\text { (68 in } 47 \text { success } \\
\text { cannulation) }\end{array}$} & $\begin{array}{l}\text { Attempts with proper } \\
\text { needle } \\
\text { average1.40(66 in } 47 \\
\text { success cannulation) }\end{array}$ \\
\hline $1^{\text {st }}$ attempt & $\begin{array}{l}68.1 \% \\
\text { (in 32 patients) }\end{array}$ & $70.2 \%$ (in 33 patients) \\
\hline $\begin{array}{l}2^{\text {nd }} \\
\text { attempt }\end{array}$ & $\begin{array}{l}23.4 \% \\
\text { (in } 11 \text { patients) }\end{array}$ & $19.2 \%$ (in 9 patients) \\
\hline $3^{\text {rd } \text { attempt }}$ & $\begin{array}{l}4.25 \% \\
\text { (in 2 patients) }\end{array}$ & $10.6 \%$ (in 5 patients) \\
\hline $4^{\text {th }}$ attempt & $\begin{array}{l}4.25 \% \\
\text { (in 2 patients) }\end{array}$ \\
\hline
\end{tabular}

\section{Complications}

\section{Table 3}

Overall complication rate $-6.25 \%$

(3 in 48 patients)

\begin{tabular}{|l|r|}
\hline $\begin{array}{l}\text { Pneumothorax } \\
(2.1 \%)\end{array}$ & 1 \\
\hline $\begin{array}{l}\text { Malposition of the catheter } \\
\text { (L/subclavian vein) }\end{array}$ & 1 \\
\hline Arterial puncture & 0 \\
\hline Haematoma/bleeding & 0 \\
\hline Hydro or haemo thorax & 0 \\
\hline Catheter related infection & 1 \\
\hline
\end{tabular}

\section{Discussion}

The subclavian vein is well suited for percutaneous access because it is related to bony structures, such as clavicle and the first rib. Subclavian catheterization was first described in 1952 by Aubaniac. (1) However, the vein is relatively far from the skin entry point and patient positioning make it less successful with $1^{\text {st }}$ attempt, repeated attempts make patients more vulnerable to develop catheter related complications. High complication rates of pneumothorax, subclavian artery puncture and malpostion of the catheter has been reported with the traditional infra clavicular approach to central venous access. ${ }^{(13)}$

In 1965 Yoffa presented the first report of supraclavicular approach to subclavian vein cannulation. ${ }^{(17)}$ Garcia et.al later fine tuned the technique to include a more shallow entry and passage of the needle. They noted that in cadaveric dissections it was simpler to aim at the junction of the subclavian vein and the internal jugular vein. Over a period of time a number of different ways, were proposed to locate the entry point, such as pocket approach by Jung et.al. ${ }^{(9)}$ Mc Donnel et al described the entry point as the junction of the medial and middle thirds of the distance between the sterno clavicular joint and the acromioclavicular joint. The advantages of the supraclavicular approach includes relatively easy surface land marks ,large target size, high overall and initial success rate ${ }^{(14)}$, successful performance despite lack of experience ${ }^{(2)}$, significant reduction in documented complications and adequate position of catheters $^{(8)(11)}$ is in line with our findings with the obese patient group. Above $90 \%$ success rate has been achieved using this technique in different patient groups such as during cardiopulmonary resuscitation $^{(3)}$, in ICU or in dialysis patients ${ }^{(2)}$, in gynaecological patients ${ }^{(6)}$ and in infants.

Yao et al reported a high tendency to damage the subclavian artery with this technique among infants ${ }^{(10)}$ and some other literature shows no superiority of one technique over the other. ${ }^{(16)}$

When it comes to anaesthetized obese patients this technique becomes more feasible due to a number of reasons. Slight trendelenburg position with head in either neutral position or slightly rotated towards the opposite side is used in this technique which provides the optimum filling of the subclavian vein. ${ }^{(4)}$ This position needs minimal interruption to the already anaesthetized patient which saves time. In some patients, 
specially in females pushing the breast and tissues away from the infraclavicular area will expose the supraclavicular area. Secondly anatomical land marks are more likely to remain constant in the supra clavicular fossa. In the absence of which clavicle comes to our aid. Compared to infraclvicular and internal jugular approaches, with this method the operator gets more space to work with. Depth at this point to the venous system is less. Therefore this provides easy passage of the catheter. The role played by the seeker needle seems questionable as the number of attempts were similar with the proper needle. In other studies too this has been the case. and this is the case with some other studies. ${ }^{(5)}$ Perhaps alteration of the patient position in-between attempts would have had some implications upon our findings. One instance we failed to site the catheter was a female of $163 \mathrm{~kg}$ with a BMI of 67 . After four supraclavicular attempts, she was subsequently catheterized using the infraclavicular approach by the consultant.

\section{Conclusion-}

Supraclavicular approach is a reliable way of obtaining central venous access with less complications in the obese.

\section{References-}

1. Aubaniac, R. (1952). "Subclavian intravenous injection; advantages and technic." Presse Med 60(68): 1456.

2. Conroy, J. M., P. R. Rajagopalan, et al. (1990). "A modification of the supraclavicular approach to the central circulation." South Med J 83(10): 1178-81.

3. Dronen, S., B. Thompson, et al. (1982). "Subclavian vein catheterization during cardiopulmonary resuscitation. A prospective comparison of the supraclavicular and infraclavicular percutaneous approaches." Jama 247(23): 3227-30.

4. Fortune, J. B. and P. Feustel (2003). "Effect of patient position on size and location of the subclavian vein for percutaneous puncture." Arch Surg 138(9): 996-1000; discussion 1001.
5. Goodwin, M. W. and C. M. Frerk (1999). "No evidence for seeker needles." Anaesthesia 54(10): 1013-5.

6. Helmkamp, B. F. and S. R. Sanko (1985). "Supraclavicular central venous catheterization." Am J Obstet Gynecol 153(7): 751-4.

7. Hind, D., N. Calvert, et al. (2003). "Ultrasonic locating devices for central venous cannulation: meta-analysis." BMJ 327(7411): 361.

8. James, P. M., Jr. and R. T. Myers (1972). "Central venous pressure monitoring: misinterpretation, abuses, indications and a new technic." Ann Surg 175(5): 693-701.

9. Jung, C. W., J. H. Seo, et al. (2007). "A novel supraclavicular approach to the right subclavian vein based on three-dimensional computed tomography." Anesth Analg 105(1): 200-4.

10. Lu, W. H., M. L. Yao, et al. (2006). "Supraclavicular versus infraclavicular subclavian vein catheterization in infants." J Chin Med Assoc 69(4): 153-6.

11. Malatinsky, J., T. Kadlic, et al. (1976). "Misplacement and loop formation of central venous catheters." Acta Anaesthesiol Scand 20(3): 237-47.

12. Mansfield, P. F., D. C. Hohn, et al. (1994). "Complications and failures of subclavian-vein catheterization." N Engl J Med 331(26): 1735-8.

13. Mitchell, S. E. and R. A. Clark (1979). "Complications of central venous catheterization." AJR Am J Roentgenol 133(3): 467-76.

14. Nevarre, D. R. and O. H. Domingo (1997). "Supraclavicular approach to subclavian catheterization: review of the literature and results of 178 attempts by the same operator." J Trauma 42(2): 305-9.

15. Randolph, A. G., D. J. Cook, et al. (1996). "Ultrasound guidance for placement of central venous catheters: a meta-analysis of the literature." Crit Care Med 24(12): 2053-8.

16. Sterner, S., D. W. Plummer, et al. (1986). "A comparison of the supraclavicular approach and the infraclavicular approach for subclavian vein catheterization." Ann Emerg Med 15(4): 421-4.

17. Yoffa, D. (1965). "Supraclavicular subclavian venepuncture and catheterisation." Lancet 2(7413): 614-7. 